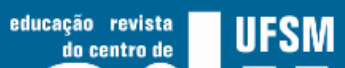

\section{Tensiones del trabajo docente en un contexto gerencialista: una revisión de literatura sobre el caso chileno}

Tensions of teaching work in a managerial context: a literature review about the Chilean case

Tensões do trabalho docente em contexto gerencial: uma revisão da literatura sobre o caso chileno

Cristian Oyarzún Maldonado

Magíster (c) en Ciencias Sociales por la Universidad de Chile, Santiago, Chile.

Rodolfo Soto González

Magíster en Psicología Educacional por la Universidad de Chile, Santiago, Chile.

Karina Moreno Díaz

Magíster en Psicología Educacional por la Universidad de Chile, Santiago, Chile.

Endereço: Alameda 2182, Santiago Centro, Chile.

E-mail: cristian.oyarzunm@usach.cl -rodolfo.soto@usach.cl - karina.moreno@usach.cl

Recebido em 21 de setembro de 2018

Aprovado em 11 de outubro de 2018

\section{RESUMEN}

Este artículo expone resultados de una revisión de literatura sobre políticas gerencialistas aplicadas a la gestión escolar y al trabajo docente en Chile. Mediante lineamientos de un tipo de revisión denominada Revisión Realista se analizaron 67 publicaciones del periodo 2003 - 2018. Los resultados muestran que este contexto institucional restringe las posibilidades de agenciamiento de los actores educativos a través de prescripciones dispuestas en variados mecanismos de estandarización y rendición de cuentas. También se reconocen tensiones del trabajo docente que surgen de desajustes entre las prescripciones del marco institucional y elementos contextuales como las concepciones locales de enseñanza y otros vinculados a la 


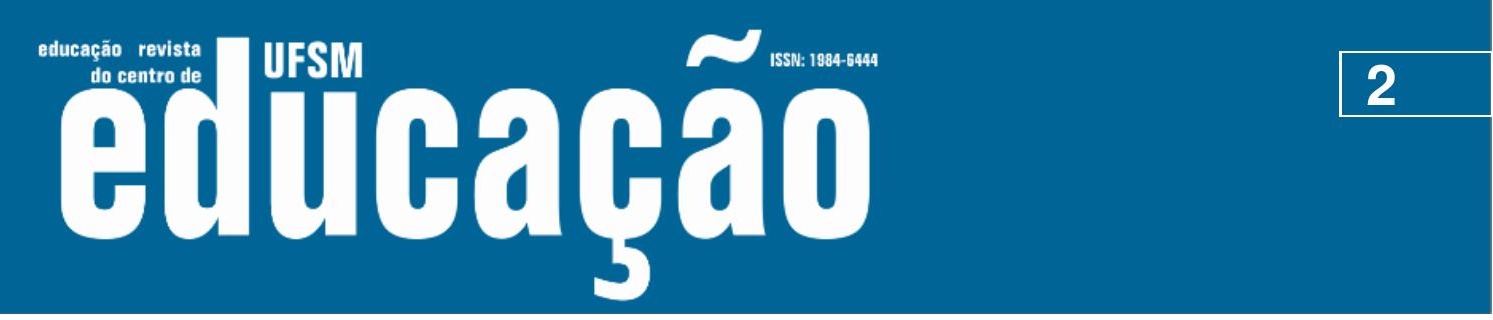

ISSN: 1984-6444 | http://dx.doi.org/10.5902/1984644434888

precarización de condiciones laborales. Estos hallazgos son discutidos en función de mejoras a la institucionalidad vigente y desafíos para la investigación.

Palabras-clave: Trabajo docente; Gerencialismo; Revisión realista de literatura.

\section{ABSTRACT}

This article presents the results of a literature review on managerial policies applied to school management and teaching work in Chile. Through guidelines of a type of revision called Realistic Review, 67 publications from the period 2003 - 2018 were analyzed. The results show that this institutional context restricts the possibilities of agency of educational actors through prescriptions arranged in various mechanisms of standardization and accountability. Teaching work tensions that arise from imbalances between the prescriptions of the institutional framework and contextual elements such as local conceptions of teaching and others linked to the precariousness of working conditions are also recognized. These findings are discussed in terms of improvements to current institutions and challenges for research.

Keywords: Teaching work; Managerialism; Realistic literature review.

\section{RESUMO}

Este artigo apresenta os resultados de uma revisão de literatura sobre políticas gerenciais aplicadas à gestão escolar e à trabalho docente no Chile. Por meio de orientações de um tipo de revisão denominada Revisão Realista, foram analisadas 67 publicações do período de 2003 a 2018. Os resultados mostram que esse contexto institucional restringe as possibilidades de agenciamento de atores educacionais por meio de prescrições organizadas em diversos mecanismos de padronização e accountability. Tensões no trabalho docente que surgem de desequilíbrios entre as prescrições do quadro institucional e elementos contextuais como concepções locais de ensino e outras vinculadas à precariedade das condições de trabalho também são reconhecidas. Essas descobertas são discutidas em termos de melhorias nas instituições atuais e desafios para a pesquisa.

Palavras-chave: Trabalho docente; Gerencialismo; Revisão realista da literatura.

\section{Introducción}

Las transformaciones económicas, sociales y culturales que experimenta una sociedad modifican las expectativas en torno a la educación, influyendo decisivamente en el diseño y funcionamiento de los sistemas escolares (MARCHESI; MARTíN, 1998). En este sentido, el campo educativo contemporáneo se ha reconfigurado en función de la globalización del capital financiero y la emergencia de una sociedad del 


\section{工 WFM entuargá}

ISSN: 1984-6444 | http://dx.doi.org/10.5902/1984644434888

conocimiento (MEJÍA, 2016), imputándosele a las escuelas y sus maestros exigencias inéditas respecto del logro de una educación de alta calidad (OCDE, 2009).

Con el objetivo de incrementar el rendimiento de los sistemas escolares, muchos diseñadores de política y tomadores de decisión han planteado que la adopción de prácticas de gestión del sector privado representa la fórmula más acertada para convertir la educación en una actividad eficiente y eficaz (BALL, 2001). De este modo, las políticas escolares de las últimas tres décadas han sido permeadas por ideas pro-privatización, que en el ámbito de la gestión educativa refieren a lo que Ball y Youdell (2008) denominan privatización endógena, es decir, en la forma de operar de las escuelas.

La concreción de esta tendencia puede encontrarse en el New Public Management o Nueva Gestión de lo Público [NGP], consistente en un programa de reforma y modernización de los servicios públicos dirigido a optimizar el logro de resultados mediante modelos de administración y gestión privados. Este paradigma de gestión incorpora una serie de medidas como competencia entre instituciones, estandarización de prácticas de desempeño, metrización de resultados, contractualización vertical con rendición de cuentas $[\mathrm{RdC}]$, consecuencias de alto riesgo, mesura en el uso de recursos, flexibilización laboral e incentivos económicos ligados a metas (ANDERSON; COHEN, 2015; HALL; MCGINITY, 2015; VERGER; NORMAND, 2015).

Como es esperable, las políticas gerencialistas modifican el quehacer de escuelas y actores educativos. Una de estas transformaciones es la redefinición de aquello que significa ser profesor y hacer pedagogía (BALL, 2003). En efecto, desde perspectivas críticas se plantea que dichas políticas aplicadas a la docencia suelen concretarse tanto en instrumentos que estandarizan el desempeño como en sistemas de evaluación vinculados a consecuencias individuales y organizacionales de alto riesgo que, en su conjunto, estructuran un régimen prescriptivo en que las prácticas de gestión y sus contenidos constituyen la única realidad posible para la docencia, excluyendo cualquier versión alternativa (BALL, 2001; MEJíA, 2006; HALL et al., 2015; HERR, 2015; TSANG; KWONG, 2016). 


\section{A Wusm Gutrathá}

ISSN: 1984-6444 | http://dx.doi.org/10.5902/1984644434888

Sin perjuicio de lo anterior, la literatura especializada señala que los sistemas escolares basados en la NGP adquieren formatos diversos, identificándose múltiples maneras de adaptación y recontextualización producto de factores sociohistóricos inherentes a cada país (HALL et al., 2015; VERGER; NORMAND, 2015; MAROY; PONS; DUPUY, 2016; HOLLOWAY; SØRENSEN; VERGER, 2017). Uno de los aspectos en que difieren estos formatos de aplicación de políticas manageriales refiere al grado de prescripción de la política y de autonomía proporcionada a los agentes educativos, encontrándose evidencia sobre dispositivos de origen gerencialista valorados favorablemente cuando confieren discrecionalidad a los actores, quienes los readaptan en función de su contexto y problemáticas locales (OLESEN; HASLE; SØRENSEN, 2016). Contrariamente, ambientes institucionales que emplean estos dispositivos condicionándolos a consecuencias de alto riesgo, socavarían las características positivas del profesionalismo, reproduciendo comportamientos orientados a salvaguardar el interés propio mediante el emprendimiento individual y el cumplimiento prescriptivo de procedimientos, en desmedro de una docencia ética, colaborativa y situada (GLEESON; KNIGHTS, 2006).

Por lo tanto, un especial interés adquieren los modelos de gestión escolar y trabajo docente que efectúan un uso irrestricto del paradigma gerencialista. Al respecto, el sistema escolar chileno sobresale por la incorporación estructural de mecanismos pro-privatización (VERGER; ZANCAJO; FONTDEVILA, 2016), tanto a nivel de la provisión como de la gestión de servicios escolares. La afirmación precedente deviene de un primer periodo de libre mercado radical instalado en los años ochenta y de un segundo momento de corrección del mercado mediante políticas gerencialistas aplicadas en los últimos quince años (FALABELLA, 2015; ASSAÉL; CORNEJO, 2018), que introdujeron un marco normativo rupturista en relación a las concepciones tradicionales de enseñanza (SISTO; FARDELLA, 2014).

Considerando lo expuesto, este artículo tiene como propósitos describir el carácter gerencialista de las políticas escolares chilenas sobre gestión y docencia, junto con identificar tensiones del trabajo docente que ocurren en dicho contexto institucional. Con este fin se realizó una revisión de literatura basada en lineamientos 


\section{THSW efituaráo}

ISSN: 1984-6444 | http://dx.doi.org/10.5902/1984644434888

de la revisión realista (PAWSON et al., 2005), que incluyó 67 publicaciones teóricas y empíricas sobre la temática. El documento se organiza en tres apartados: el primero describe la metodología, específicamente, define la revisión realista de literatura y las tensiones como herramienta analítica; en el segundo se exponen los resultados sobre el contexto institucional y las tensiones; finalmente, el tercer apartado de discusión y conclusiones se desarrolla en función del mejoramiento de las políticas sobre docencia y los desafíos para la investigación empírica.

\section{Procedimientos metodológicos}

Se emplearon lineamientos de la revisión realista, la cual es un tipo de revisión de literatura propuesta por Pawson et al. (2005), que consiste en recopilar, analizar y sintetizar la producción académica sobre un programa de intervención social o política pública a objeto de comprender las relaciones entre el contexto, sus mecanismos y resultados. Con más detalle, la revisión realista es pertinente para examinar el modo en que los factores contextuales pueden mediar o influir en la aplicación de una política pública o una intervención social, contrastando, a partir de la evidencia recogida, sus propósitos teóricos con los efectos que experimentan quienes las implementan y/o utilizan.

En concordancia con la lógica de la revisión realista, los resultados son expuestos en dos núcleos. El primero corresponde a la descripción del contexto gerencialista en cuanto a sus principios teóricos y dispositivos presentes en la política escolar chilena; mientras en el segundo se presentan dos tensiones sustentadas en el análisis y contrastación de la literatura. Cabe mencionar que las tensiones, en tanto herramienta analítica, son utilizadas habitualmente en el campo de la investigación de políticas educativas en Chile, no obstante, existen escasas delimitaciones conceptuales sobre ellas. En este sentido, las tensiones pueden definirse como un estado de presión ambivalente, ejercido sobre los agentes sociales por un contexto institucional que dispone de un doble mensaje normativo y contradictorio (COHEN; MOFFITT, 2009). En consecuencia, esta herramienta permite visualizar contradicciones e incoherencias del diseño institucional que enmarca el quehacer cotidiano de los actores educativos. 


\section{A Wusm entioará}

ISSN: 1984-6444 | http://dx.doi.org/10.5902/1984644434888

En términos procedimentales, el primer criterio que guio la pesquisa fue la consulta en bases de datos como Education Research Information Center, Web of Science, Scopus, Dialnet y Scielo, usando las combinaciones - y su símil en inglés de palabras clave: trabajo docente Chile, profesión docente Chile, política docente Chile y gerencialismo docencia Chile. Un segundo criterio de selección/exclusión de los artículos correspondió al lapso temporal de su publicación, ubicándose éste, primordialmente, en el periodo 2003 - 2018, pues como adelantamos constituye el lapso de consolidación y mayor debate académico sobre las políticas de gestión escolar y docencia.

Considerando que el tema de política docente convoca diversos grupos de interés y múltiples perspectivas, como centros de investigación y agrupaciones sociales, se optó por incorporar publicaciones emitidas por instituciones vinculadas al área con el objetivo de evitar "fugas de literatura" y dotar de integralidad la revisión, como MIDE UC y otras provenientes de colectivos sociales. El resultado definitivo del trabajo es un total de 67 publicaciones que se disgregan de la siguiente forma: 43 artículos empíricos y 24 análisis teóricos sobre gestión escolar y docencia en Chile. Entre el material empírico se identifican 31 estudios cualitativos, 7 cuantitativos, 3 etnografías y 2 mixtos. Adicionalmente, se revisó la reciente ley que Crea el Sistema Nacional de Desarrollo Profesional Docente [ley de Carrera Docente]. Si bien, la propuesta original de Pawson et al. (2005) se aplica a la síntesis de hallazgos de estudios primarios, en esta ocasión fueron incorporados análisis teóricos y la ley citada a fin de robustecer la caracterización del marco institucional estudiado.

\section{Resultados}

\section{La NGP en la política sobre gestión escolar}

La privatización de la educación escolar chilena tiene un momento de instalación y otro de consolidación. El primero ocurrió durante la dictadura cívicomilitar en la década de los ochenta, cuando se introdujeron mecanismos de mercado como el subsidio per cápita, la libre elección y la incorporación de proveedores privados. Simultáneamente, las responsabilidades del Estado fueron reducidas drásticamente a través de un proceso de descentralización administrativa de las 


\section{工 WFM Euflaaráo

ISSN: 1984-6444 | http://dx.doi.org/10.5902/1984644434888

escuelas públicas, desde el Ministerio de Educación [MINEDUC] a municipios comunales, así como por la pérdida de facultades regulatorias en la formación y el trabajo docente, también entregados a la regulación del mercado (VERGER et al., 2016).

El segundo momento acontece en la primera década del siglo XXI, cuando progresivamente se instituyen políticas basadas en la NGP. Primordialmente, estas políticas han intentado reorientar el sistema hacia una mayor eficiencia por medio de estándares de desempeño y resultados, instancias periódicas de $\mathrm{RdC}$ e incentivos, medidas aplicadas tanto a nivel de la gestión organizacional de las escuelas como del desempeño individual de los profesionales de la educación (FALABELLA, 2015; CORNEJO et al., 2015). De manera más puntual y a diferencia de otros países, en Chile las políticas gerencialistas aparecen como una forma de corregir las imperfecciones y desigualdades generadas por la reforma radical de mercado a través de la figura de un Estado Evaluador (VERGER; NORMAND, 2015; HOLLOWAY et al., 2017; PARCERISA; FALABELLA, 2017). Efectivamente, las persistentes críticas endosadas a la educación escolar desde fines los noventa, ya sea por sus exiguos resultados en términos de calidad educativa o por el creciente malestar social entorno a ésta (CABALIN, 2012), movilizaron a las autoridades políticas para introducir reformas gerencialistas revestidas de una retórica que las posicionaba como la mejor forma para incrementar la calidad del sistema (SISTO; FARDELLA, 2011).

En este contexto, se identifican dos hitos que marcan el ingreso de la NGP a gran escala en la política escolar chilena. Uno de ellos es la ley de Subvención Escolar Preferencial [SEP] de 2008, consistente en un subsidio incrementado para la atención de estudiantes en condición de pobreza, cuya entrega está condicionada por mecanismos verticales de contractualización y accountability académico (ROJAS; LEYTON, 2014; LIGUEÑO; PALACIOS; SANDOVAL, 2018). En lo específico, esta ley conlleva que los administradores de los centros escolares suscriban un convenio con el MINEDUC, el cual fija metas auditables de desempeño académico y establece la obligatoriedad de invertir los recursos adicionales en un "plan de mejora" basado en dimensiones y prácticas de gestión estandarizadas $(\mathrm{OPECH}, 2009)$. 


\section{OF WF Gutrahâ}

ISSN: 1984-6444 | http://dx.doi.org/10.5902/1984644434888

Otro hito es el fortalecimiento de estos dispositivos mediante la instalación de un Sistema de Aseguramiento de la Calidad de la Educación [SAC] promulgado en 2011. EI SAC crea dos instituciones, una Superintendencia que fiscaliza y sanciona el cumplimiento normativo, y una Agencia de Calidad destinada a evaluar el desempeño y publicar rankings de resultados respecto de un conjunto de políticas previas como el Sistema de Medición de Calidad de la Enseñanza y la misma SEP, contemplándose el cierre definitivo de las escuelas que mantienen un rendimiento insuficiente durante cuatro años. Todo lo dicho contribuiría a consolidar una concepción meramente instrumental de la enseñanza, en que predomina la consecución de resultados académicos como eje articulador sistema (FALABELLA; OPAZO, 2014; FALABELLA, 2016; PINO; OYARZÚN; SALINAS, 2016; PARCERISA; FALABELLA, 2017).

Diversos análisis críticos de documentos oficiales y leyes han reconocido características gerencialistas en otros marcos normativos contemporáneos a los descritos. Tal es el caso de la ley de Calidad y Equidad de la Educación de 2011, que incrementó los mecanismos de evaluación para el personal docente y flexibilizó sus condiciones laborales con incentivos económicos de carácter individual vinculados a metas, así como también asignó un rol gerencial a los directores de escuela mediante convenios de desempeño (CORNEJO et al., 2015; BISCARRA; GIACONI; ASSAÉL, 2015; ASSAÉL; CORNEJO, 2018).

De este modo, las políticas gerencialistas han rediseñado la relación entre las escuelas y el Estado, configurándose un modelo de mercado performativo (FALABELLA, 2016) con doble rendición de cuentas - hacia el mercado y hacia el Estado - (WEINSTEIN et al., 2016), en que los establecimientos logran subsistir solo cuando reproducen parámetros de desempeño definidos y controlados por entidades estatales. En consecuencia, deviene la necesidad de adscribir al modelo de gestión prescrito, lo cual transforma el trabajo de los profesionales de la educación en un quehacer condicionado por dispositivos externos que operan mediante la interacción simultanea de metas, evaluaciones, distintas formas de RdC, sanciones y asignaciones salariales por desempeño (CARRASCO, 2013). 


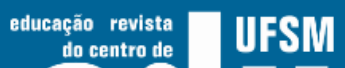 Eulloâ̧a}

ISSN: 1984-6444 | http://dx.doi.org/10.5902/1984644434888

\section{Gerencialismo en el trabajo de los profesores chilenos}

La trayectoria de la política magisterial reciente evidencia dos lógicas, la lógica de la recuperación y la lógica del desempeño (SISTO; FARDELLA, 2014). La primera se concretizó con la promulgación del Estatuto Docente de 1991, que representa una medida compensatoria ante la precarización extrema de las condiciones laborales cometida en dictadura. El estatuto incluyó mejoras para los profesores municipales, fijando un piso salarial llamado "renta básica mínima nacional", un sistema de calificaciones, asignaciones por antigüedad y perfeccionamiento, entre otras medidas (BONIFAZ, 2011). Por su parte, la lógica del desempeño comienza a fines de la década de los noventa, con el propósito tanto de superar limitaciones en los procedimientos evaluativos del Estatuto Docente, predominantemente administrativos, como de suplir la falta de fuentes de información fiables para retroalimentar el desempeño del profesorado, en ese momento cuestionado por los deficientes resultados de aprendizaje (ÁVALOS; ASSAÉL, 2006).

Para subsanar esta coyuntura, se conformó una comisión integrada por representantes del gremio docente, empleadores del sector municipal y técnicos del MINEDUC (ÁVALOS, 2004). Este trabajo generó propuestas sobre un instrumento de estándares de desempeño, posteriormente denominado Marco para la Buena Enseñanza [MBE], y un modelo de evaluación formativa destinado a profesores del sector municipal. En este escenario surgió la noción en torno a la construcción del sistema evaluativo, pues el MBE fue sometido a dos consultas magisteriales y, además, se efectuó una votación que congregó a más del $80 \%$ de los profesores municipales, quienes aprobaron el sistema con un $63 \%$ de adhesión (ASSAÉL; PAVEZ, 2008; BONIFAZ, 2011). Empero, análisis ulteriores reconocen que dicho acuerdo tuvo bases políticas frágiles, que decantaron en la formación de expectativas disimiles acerca de los objetivos y la utilidad de la evaluación para los grupos interesados (TAUT et al., 2010). De este modo, la implementación del sistema y sus sucesivas modificaciones generaron un escenario ambiguo, con elementos formativos representados en las oportunidades de perfeccionamiento y otros de corte sumativo o de RdC, como el cese laboral por bajo desempeño (ÁVALOS, 2017). 


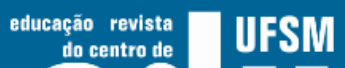 Eulloahá}

ISSN: 1984-6444 | http://dx.doi.org/10.5902/1984644434888

En efecto, el sistema promulgado en 2003 se convirtió en una evaluación basada en estándares y ligada a consecuencias. El formato incorporó cuatro instrumentos para evaluar el grado de ajuste a las prácticas estandarizadas delMBE, estableciéndose también cuatro categorías correlativas para clasificar el desempeño docente (FLOTTS; ABARZÚA, 2011; TAUT; SUN, 2014). Entonces, aquellos calificados en nivel destacado podían optar, voluntariamente y previa aprobación de una prueba técnica, a un incentivo económico denominado Asignación Variable por Desempeño Individual [AVDI] e integrarse a la Red Maestros de Maestros, mientras quienes estuviesen en nivel insatisfactorio durante tres evaluaciones consecutivas debían salir del sistema. Posteriormente, el régimen de evaluaciones y consecuencias de alto riesgo se intensificó con la ley de Calidad y Equidad, que aumentó la frecuencia de las evaluaciones y redujo a dos las oportunidades para superar el nivel insatisfactorio (BONIFAZ, 2011).

Actualmente, el sistema de evaluación con incentivos y sanciones permanece vigente, a pesar de la ley de Carrera Docente (2016). Este marco incorporó cambios relevantes, como la noción de carrera profesional asociada a mejoras salariales (RUFFINELLI, 2016). Sin embargo, mantuvo la matriz evaluativa descrita $y$, adicionalmente, vinculó los progresos de carrera a un nuevo sistema que evalúa la adecuación al MBE mediante un portafolio y una prueba técnica de índole curricular (CHILE, 2016).

En suma, las recientes modificaciones aportan a la consolidación de un contexto gerencialista que, fundamentalmente, enfatiza en la RdC individual. En esta línea, Cavieres y Apple (2016) sostienen que la nueva ley docente intenta difundir un "buen sentido común", en donde la estandarización y la medición constituyen los principales mecanismos para inducir el desarrollo profesional de los profesores. Desde una perspectiva teórica distinta, Ávalos (2017) resalta que el modelo de evaluación aun dispone de elementos sumativos que tensionan toda intención formativa $y$, además, incluye medidas con escaso respaldo empírico, como la inusitada importancia atribuida a la prueba de conocimientos curriculares y sus consecuencias. Esto último coincide con el informe de la OCDE (2017), el cual indica que los profesores perciben poca utilidad formativa respecto de los mecanismos de 


\section{工 Hsy

ISSN: 1984-6444 | http://dx.doi.org/10.5902/1984644434888

retroalimentación contemplados en la evaluación.

Considerando todo lo expuesto y a excepción del sistema de evaluación de 2003, el contexto gerencial ilustrado representa un caso de reforma arriba-abajo, desprovisto de legitimación social. Como muestra, se tiene que el informe técnico que fundamenta el SAC solo fue concordado con los conglomerados políticos dominantes, siendo rechazado por el "Bloque Social", un colectivo que representaba a estudiantes, profesores y apoderados (PARCERISA; FALABELLA, 2017). Por su parte, la tramitación de la ley de Carrera Docente estuvo antecedida de múltiples movilizaciones, que culminaron con una paralización del profesorado que se extendió por más de 50 días (ASSAÉL; CORNEJO, 2018).

Resumiendo: las políticas chilenas sobre gestión escolar y docenciareproducen de manera fidedigna los principios de la NGP, configurando un ambiente institucional que prepondera medios exógenos, como la estandarización y los mecanismos de control, para asegurar la enseñanza, minimizando la autonomía y capacidades profesionales de los agentes educativos. La tabla 1 expone los principios gerencialistas y su concreción en la política chilena. 


\section{Tosm Eltoará}

ISSN: 1984-6444 | http://dx.doi.org/10.5902/1984644434888

Tabla 1 - Principios y dispositivos gerencialistas presentes en la política escolar chilena.

\begin{tabular}{l|l}
\hline Principios NGP & Dispositivos de la política escolar chilena \\
\hline Metrización de resultados & $\begin{array}{c}\text { Medición estandarizada de aprendizajes } \\
\text { mediante el SIMCE }\end{array}$ \\
\hline Competencia entre instituciones & $\begin{array}{c}\text { Financiamiento per cápita vía vouchery } \\
\text { publicación de ranking de desempeño de } \\
\text { escuelas según SIMCE }\end{array}$ \\
\hline $\begin{array}{l}\text { Contractualización vertical con RdC } \\
\text { externa y consecuencias de alto riesgo }\end{array}$ & $\begin{array}{c}\text { Convenio Estado-escuela por SEP y cierre } \\
\text { de escuelas por desempeño insuficiente en } \\
\text { cuatro años correlativos }\end{array}$ \\
\hline $\begin{array}{l}\text { Evaluación docente asociada a } \\
\text { incentivos económicos individuales }\end{array}$ & \begin{tabular}{c} 
AVDI, carrera ligada a aumento salarial \\
\hline $\begin{array}{l}\text { Estandarización de prácticas de } \\
\text { desempeño }\end{array}$
\end{tabular} \\
\hline $\begin{array}{l}\text { Flexibilización laboral y mesura en el } \\
\text { uso de recursos }\end{array}$ & $\begin{array}{c}\text { Montratación transitoria de profesionales de } \\
\text { la educación con recursos SEP de gestión estandarizadas }\end{array}$ \\
\hline
\end{tabular}

Fuente: Elaboración propia en base revisión de literatura

\section{Tensiones de la docencia en un contexto gerencialista}

La institucionalidad descrita produce tensiones para la docencia que surgen por efecto de mensajes contradictorios en los marcos normativos y/o de elaboraciones sociohistóricas y técnicas opuestas que coexisten con el discurso hegemónico. Se identifican dos tensiones:

1. Principal responsable del éxito escolar versus Actor social históricamente precarizado;

2. Ejecutor de prácticas prescritas versus Profesional reflexivo que crea prácticas situadas.

La primera tensión alude a que los marcos gerencialistas, en tanto 


\section{WEM Gutrathá}

ISSN: 1984-6444 | http://dx.doi.org/10.5902/1984644434888

elaboraciones discursivas de la voz oficial del Estado, ubican al profesor como principal responsable y eje articulador del éxito educativo. Sin embargo, latrayectoria de las políticas exhibe una pauperización general de las condiciones, materiales e inmateriales, del trabajo docente. Lo anterior decanta en un desequilibrio entre exigencias y condiciones necesarias para un trabajo de elevada complejidad.

Efectivamente, investigaciones basadas en análisis de documentos oficiales y entrevistas a docentes, concuerdan que las políticas vigentes interpelan la efectividad del profesorado y le asignan centralidad absoluta en el logro de mejores resultados (ÁVALOS; SOTOMAYOR, 2012; SISTO; FARDELLA, 2011; FARDELLA; SISTO, 2013; REYES et al., 2010; SOTO et al., 2016; ETCHEBERRIGARAY et al., 2017; FERNÁNDEZ, 2018). En este sentido, Fernández (2018) reportó que documentos del MINEDUC atribuyen la brecha de rendimientos entre estudiantes de distintos grupos

socioeconómicos a la baja calidad de la docencia, sin contemplar explicaciones multicausales y factores sociales. Complementariamente, Santori (2016) identificó una proliferación de publicaciones sobre éxito educativo en sectores vulnerables, que refuerzan la vinculación entre docentes efectivos y mejores resultados, instalando una noción de "Yes, we can!" sustentada solo en el voluntarismo y la capacidad individual.

Por su parte, aun cuando la ley de Carrera Docente es considerada un cambio desde la precarización sistemática a los logros (RUFFINELLI, 2016), estas modificaciones son recientes y no superan todas las limitaciones que detalla la literatura. Entre los aspectos abordados por esta ley se hallan incrementos remuneracionales y regulaciones en la formación que intentan morigerar la fuerte devaluación del estatus social de la docencia (BELLEI; VALENZUELA, 2010, BELLEI; VALENZUELA 2013) y la desigual distribución de profesores en función del nivel de su formación inicial y posterior incorporación al mercado laboral (MECKES; BASCOPÉ, 2012; RIVERO, 2015; RUFFINELLI; GUERRERO, 2009; RUFFINELLI, 2014). No obstante, estos avances evidencian que la problemática docente es concebida únicamente en torno a factores económicos y de adquisición de conocimientos, omitiéndose otros factores materiales e inmateriales igualmente importantes. 


\section{A Wusm Gutrahá}

ISSN: 1984-6444 | http://dx.doi.org/10.5902/1984644434888

Uno de los aspectos pendientes es la inadecuada distribución del tiempo lectivo/no lectivo y sus consecuencias (FORO POR EL DERECHO A LA EDUCACIÓN CHILE, 2015). Los datos plantean que los profesores chilenos trabajan en promedio 29 horas semanales, de estas 27 son lectivas, reduciéndose drásticamente su tiempo para desarrollar actividades de planificación y trabajo colegiado (OCDE, 2017). En esta línea, Cabezas et al. (2017) presentan evidencia negativa y robusta acerca de la asociación entre horas extra-laborales y satisfacción laboral, la que favorecería el burnout y los efectos de sobrecarga. Esto concuerda con investigaciones previas, como la de Cornejo (2009) que en base a una muestra representativa de profesores de Santiago presentó hallazgos sobre altos niveles de burnout, sobrecarga horaria, sobre exigencia ergonómica y precariedad de materiales; mientras Cuadra et al. (2015) encontraron que desde la perspectiva de los docentes su salud laboral mejoraría con la disminución/aumento de horas de trabajo lectivo/no lectivo, la erradicación de presiones por resultados y reconocimientos al trabajo docente.

Esta tensión se transfiere a la cotidianidad de los profesores. Al respecto, la

interacción de las distintas políticas descritas, como por ejemplo la ley SEP, configuraría un relato que posiciona a los profesores como sujetos en permanente

falta, culpabilizándolos ante las dificultades que devienen de hacerse cargo individualmente de la desigualdad escolar, pues la lógica del management solo entrega reconocimiento ante la obtención de resultados medibles (ROJAS; LEYTON, 2014; GUERRERO, 2017). Por su parte, las etnografías revisadas plantean que la

exacerbada preocupación por resultados induce prácticas discriminatorias para mejorar la composición social y académica de las escuelas, a la vez que en contextos vulnerables y de bajo desempeño genera un sentimiento de incapacidad e impulsa los esfuerzos individuales hacia la producción de evidencia para la $\mathrm{RdC}$, que no se traduce en mejoras (ACUÑA et al., 2014; ASSAÉL et al., 2012; ASSAÉL et al., 2014). La segunda tensión refiere a la progresiva estandarización de la labor pedagógica en función de prácticas y resultados preestablecidos externamente (ASSAÉL; CORNEJO, 2018; ASSAÉL; ALBORNOZ; CARO, 2018; SISTO; FARDELLA, 2014). El carácter exógeno de las prescripciones que fijan prácticas y metas, como "manuales de mejores prácticas" y "estándares de aprendizaje", conlleva 


\section{N Wsm Eutioará}

ISSN: 1984-6444 | http://dx.doi.org/10.5902/1984644434888

suponer que los docentes carecen de capacidades para tomar decisiones educativas, de manera que estas herramientas ofrecen un repertorio de acciones que puede reemplazar el "buen juicio profesional” de los maestros (CARRASCO, 2013).

Relacionado con lo anterior, Fernández et al. (2016) plantean que el marco imperante subordina el trabajo de los docentes, ubicándolos "en una posición pasiva respecto al acto educativo y en una función meramente técnica como ejecutores de contenidos curriculares definidos previamente y sin su participación" (p. 298). En la misma línea, Cavieres y Apple (2016) afirman que la ley de Carrera Docente es la elaboración de una elite tecnocrática, que incluyó espacios muy limitados de participación para el magisterio, siendo inhabilitado de incorporar prerrogativas que representen sus saberes y necesidades.

Lo señalado concuerda con la perspectiva de los profesores, quienes experimentan e interpretan las prescripciones sobre su trabajo como un esfuerzo institucional para modelar sus prácticas locales desde referentes externos que desconocen su quehacer cotidiano, el que, desde su punto de vista, constituye la verdadera pedagogía (FARDELLA; SISTO, 2015). Con más detalle, Sisto (2012) señala que "parte significativa de las valoraciones negativas contra la política se liga precisamente a descripciones de esta en tanto construida por personas que no conocen "la realidad educativa", por lo cual se la valora como no pertinente" (p. 41).

En un sentido semejante, una investigación basada en 60 entrevistas a profesores exploró esta dicotomía entre saberes locales y estándares predefinidos (SISTO, 2011). Los resultados reafirmaron la idea que los maestros están en una posición de acatamiento en relación a los contenidos de la política evaluativa, que catalogan como "subjetivos" en tanto conocimientos arbitrarios elaborados por "expertos". En oposición, el colectivo docente reivindica su propio saber, el que diferencian de los estándares y consideran como atingente al contexto social de sus estudiantes. El aspecto recién mencionado, el contexto, es relevado en la investigación de Reyes y Akkari (2017), quienes encontraron que, en sectores de Santiago caracterizados por pobreza y violencia urbana, el discurso de los docentes disiente de las nociones de calidad y de las prescripciones emanadas desde las políticas, tendiendo a expresar concepciones hibridas de la enseñanza que incorporan 


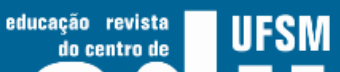 entioará}

ISSN: 1984-6444 | http://dx.doi.org/10.5902/1984644434888

la atención de necesidades psicosociales del estudiantado como un indicador de calidad tanto o más importante que los resultados académicos.

De forma concordante con lo anterior, Ferrada (2017) presentó resultados de un estudio realizado a partir de 16 grupos focales que incorporaron perspectivas de 68 profesores pertenecientes a colegios públicos y privados de Santiago. Reportándose que, si bien los maestros asocian su trabajo a una extensa multiplicidad de bienes o propósitos pedagógicos, como por ejemplo la afectividad de los estudiantes o la capacidad de colaboración entre pares, las actuales políticas orientarían su quehacer únicamente hacia una noción estrecha de enseñanza basada en resultados cuantificables, debiendo renunciar a las prácticas pedagógicas que consideran justas o distanciarse de los lineamientos de las políticas para de ese modo preservar su ethos profesional. Estos hallazgos remiten a las dificultades de prescribir y estandarizar la docencia, pues como explica Cornejo (2017) en esta actividad existe una distancia importante entre lo prescrito y lo real, pues ante todo se trata de una praxis situada y vincular.

Como se adelantó la adscripción al modelo está constreñida o condicionada intensamente por dispositivos de RdC e incentivos individuales. Los mecanismos de control ejercen un fuerte impulso hacia la reproducción de estándares y el logro de resultados, lo que colisiona con identidades tradicionales, más ligadas al servicio social y al sentido moral de la enseñanza (NÚÑEZ, 2007; SISTO, 2012). El estudio de Quaresma y Orellana (2016) ilustra esta disputa, reportando que profesores de escuelas públicas de alto desempeño perciben que el énfasis en la RdC carece de sentido para ellos y no guía sus convicciones profesionales. Por su parte, el uso de incentivos, mayormente individuales, y el pago por mérito, aparecen como elementos trascendentales de las políticas docentes para optimizar desempeños, tanto a nivel local como nacional (SISTO; MONTECINOS; AHUMADA, 2013; ACUÑA, 2015), empero la evidencia no ha constatado su real efectividad (GALLEGO et al., 2008; LARA; MIZALA; REPETTO, 2010) y ejercen un influjo contrario a las elaboraciones que resaltan la importancia del Capital Social docente y el trabajo colegiado (MONTECINOS et al., 2014; OCDE, 2017). 


\section{A Wusm Gutrathá}

ISSN: 1984-6444 | http://dx.doi.org/10.5902/1984644434888

Por último, la noción de docente como ejecutor de prácticas prefabricadas rigidiza el acto educativo, siendo escasamente atingente a los requerimientos actuales de la enseñanza, caracterizada por una creciente diversidad del estudiantado en cuanto a sus necesidades de aprendizaje y su reposicionamiento como sujeto social. Considerando esto, se plantea que la lógica estandarizadora, centrada en prácticas homogéneas y obtener resultados medibles, obstaculiza las perspectivas inclusivas que conciben la atención de la diversidad como la estrategia más adecuada para lograr aprendizajes en la mayoría de los educandos y no en un grupo delimitado (MUÑOZ; LÓPEZ; ASSAÉL, 2015).

\section{Discusión y conclusiones}

El presente artículo nos ha permitido revisar la literatura sobre el contexto institucional reciente de la gestión escolar y la docencia en Chile. Como corolario de este trabajo se desprende un primer hallazgo: este caso nacional representa una aplicación fidedigna de principios y dispositivos propios de la NGP, que sobresale por el uso de mecanismos de estandarización y control para alcanzar mejoras en los desempeños y resultados de su sistema escolar. Efectivamente, el análisis realizado evidencia la centralidad que adquieren en este contexto la estandarización, como medio para asegurar que el trabajo docente reproduzca prácticas y resultados prescritos, y, junto con ella, la utilización de distintas formas de RdC con consecuencias de alto riesgo e incentivos económicos mayormente individuales, que intentan promover la adscripción al modelo propuesto por la política, aun cuando existe poca o ninguna evidencia clara sobre su real efectividad.

En la sección introductoria planteamos que la adopción de políticas gerencialistas presentaba variaciones, encontrándose formatos de esta política que disponen de diferentes grados de autonomía y prescripción, lo que da origen a valoraciones y resultados disimiles. En este sentido, el primer hallazgo ubica el diseño institucional chileno en un formato de NGP altamente prescriptivo y fuertemente restrictivo de la autonomía profesional de los agentes educativos. Ambos aspectos resultan temas recurrentes en parte importante de la literatura pesquisada (v.g. ASSAÉL et al., 2012; CARRASCO, 2013; CORNEJO et al., 2015), propiciando el 


\section{Fusm Gutrathá}

ISSN: 1984-6444 | http://dx.doi.org/10.5902/1984644434888

riesgo de reproducir comportamientos performativos dirigidos a resguardar intereses personales mediante el emprendimiento individual y acciones orientadas a "cumplir por cumplir".

Por su parte, problematizar la gestión escolar y el trabajo docente a partir de tensiones nos ha posibilitado cuestionar el "buen sentido común" que intentan instalar los marcos regulatorios gerencialistas y también reconocer sus contradicciones. En base a esto, el segundo hallazgo de esta revisión alude a lo siguiente: las condiciones institucionales prescritas en la política presentan una brecha significativa con las necesidades de los agentes educativos y con las medidas técnicas sugeridas por la evidencia empírica.

Considerando ambos resultados de la revisión, uno de los factores que parece clave en el mejoramiento de las condiciones institucionales corresponde a la generación de espacios de participación deliberativos, que permitan a los profesores incidir en la construcción de los marcos regulatorios de su trabajo. Las investigaciones analizadas señalan que los docentes perciben las políticas como elaboraciones de "expertos", ajenas a "la realidad educativa" y que los incentivos no se ajustan al sentido ético que ellos otorgan a su profesión. Paralelamente, estas políticas exhiben una trayectoria marcada por una exigua legitimación social.

En consecuencia, si en algún momento se habló de una "agenda pendiente" en la educación chilena, esta debiese considerar la creación de instancias que hagan parte de los procesos decisionales al gremio docente, incorporando de forma simétrica sus propuestas técnicas, necesidades profesionales y concepciones de la enseñanza. Cabe mencionar que, esta conjetura no implica desacreditar la evidencia científica que sustenta, por ejemplo, los modelos universales de buenas prácticas, sino desarrollar espacios dialógicos en que las normas y los estándares sean definidos de maneratal que puedan ser interpretados y adaptados de forma crítica por los agentes educativos "en" y "para" sus contextos.

En el ámbito de la investigación son variadas las proyecciones que surgen a partir de este artículo. Al respecto, resulta relevante tener en cuenta que el diseño e implementación de políticas públicas no es un proceso mecánico, pues se encuentra mediatizado por diversas variables sociohistóricas y subjetividades condicionadas por 


\section{त Fism entuahá}

ISSN: 1984-6444 | http://dx.doi.org/10.5902/1984644434888

múltiples grupos de interés. La comprensión de dichas subjetividades y su pluralidad de expresiones implica el desarrollo de dispositivos de investigación de orden cualitativo.

En este sentido, una línea de investigación posible apunta a indagar en los modos que docentes, de aula y directivos, recontextualizan, esto es interpretan y actúan, las políticas gerencialistas y sus dispositivos concretos. Si bien, la pesquisa arrojó 31 estudios cualitativos, estos en general no capturan la multiplicidad de interpretaciones y lógicas de acción que pueden desencadenarse en el contexto escolar, sino que se focalizan en temas puntales. Específicamente, se tiene que una parte de estas investigaciones se aboca al análisis crítico de leyes y otra recoge significaciones del profesorado, pero principalmente en relación con el sistema de evaluación de desempeño previo a las modificaciones introducidas por la ley de Carrera Docente.

En relación con lo anterior, la etnografía aparece como la metodología más pertinente para avanzar en el entendimiento de los complejos procesos de recontextualización que tienen lugar en el cotidiano de la escuela. Sin duda este es un desafío para la investigación, ya que a la luz de nuestra revisión los métodos etnográficos han sido escasamente utilizados para investigar los efectos que generan los marcos regulatorios gerencialitas, encontrándose apenas tres estudios de este tipo. Una ventaja que supone el uso de esta metodología refiere a la posibilidad que otorga para identificar y comprender con mayor claridad las diversas formas de recontextualización posibles en el ambiente escolar, las que pueden abarcan respuestas tan disimiles como la resistencia activa, la elaboración de formas pedagógicas alternativas o la adscripción sin compromiso emocional a las políticas, aspectos en los que la literatura pesquisada no es del todo concluyente aún.

Finalmente, la revisión puso de manifiesto la precarización de las condiciones del trabajo docente en Chile, y en particular la forma en que elementos del contexto institucional pueden afectar dimensiones inmateriales del trabajo de los profesores (v. g. distribución de tiempo lectivo/no lectivo y salud laboral). Más detalladamente, solo se registraron tres investigaciones indexadasque abordaban la temática (CORNEJO, 2009; CUADRA et al., 2015; CABEZAS et al., 2017). Por lo tanto, es una proyección 


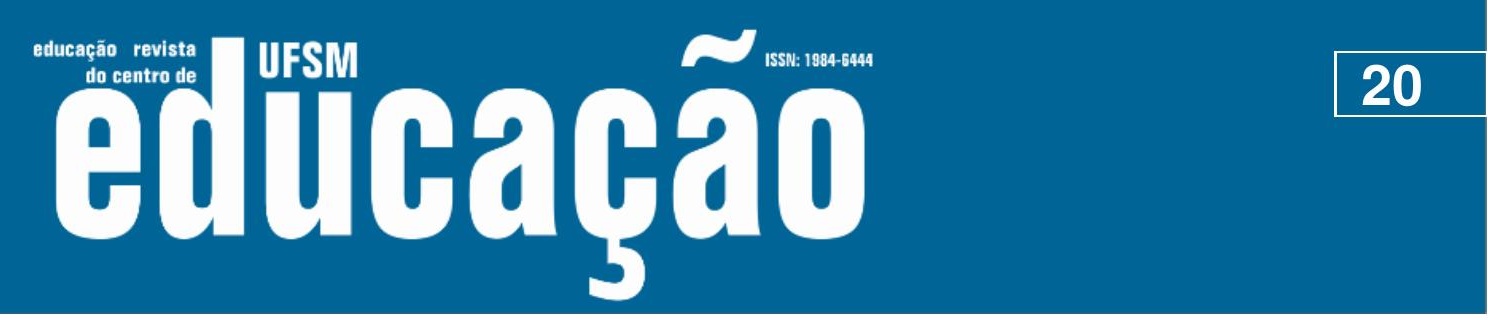

ISSN: 1984-6444 | http://dx.doi.org/10.5902/1984644434888

para la investigación científica explorar los potenciales efectos nocivos de las reformas gerencialistas aplicadas en el trabajo pedagógico, por ejemplo, en cuanto al impacto de las presiones por resultados y su lógica productivista en la emocionalidad de los profesores, aspecto relevante si considera, como hemos reiterado, que se trata de un trabajo eminentemente vincular y afectivo.

\section{Referencias}

ACUÑA, F. Incentivos al trabajo profesional docente y su relación con las políticas de evaluación e incentivo económico individual. Estudios pedagógicos, v. 41, n.1, p. 7-26, 2015.

ACUÑA, F.; ASSAÉL, J.; CONTRERAS, P.: PERALTA, B. La traducción de los discursos de la política educativa en la cotidianeidad de dos escuelas municipales chilenas: La metáfora médica como vía de análisis. Psicoperspectivas, v. 13, n. 1, p. 46-55, 2014.

ANDERSON, G.; COHEN, M. I. Redesigning the identities of teachers and leaders: A framework for studying new professionalism and educator resistance. Education Policy Analysis Archives, v. 23, n. 85, 2015.

http://dx.doi.org/10.14507/epaa.v23.2086

ASSAÉL, J.; CORNEJO, R. Work regulations and teacher subjectivity in a context of standardization and Accountability Policies in Chile. In: NORMAND, R.; LIU, M.; CARVALHO, L.; ANDRADE, D.; LEVASSEUR, L. (Eds.). Education Policies and the Restructuring of the Educational Profession. Beijing: Springer, 2018. p. 245257.

ASSAÉL, J.; PAVEZ, J. La construcción e implementación del Sistema de Evaluación del Desempeño Docente Chile: principales tensiones y desafíos. Revista Iberoamericana de Evaluación Educativa, v. 1, n. 2, p. 42-55, 2008.

ASSAÉL, J.; ALBORNOZ, N.; CARO, M. Estandarización educativa en Chile: tensiones y consecuencias para el trabajo docente. Educação Unisinos, v. 22, n. 1, p. 83-90, 2018.

ASSAÉL, J.; ACUÑA, M.; CONTRERAS, P.; CORBALÁN, F. Transformaciones en la cultura escolar en el marco de la implementación de políticas de accountability en Chile. Un estudio etnográfico en dos escuelas clasificadas en recuperación.

Estudios Pedagógicos, v. 40, n. 2, p. 7-26, 2014.

ASSAÉL, J.; CONTRERAS, P.; CORBALÁN, F.; PALMA, E.; CAMPOS, J.; SISTO, V.; REDONDO, J. Ley SEP en escuelas municipales emergentes: ¿cambios en la identidad docente? Revista de Pedagogía Crítica, v. 11, p. 219-228, 2012. 


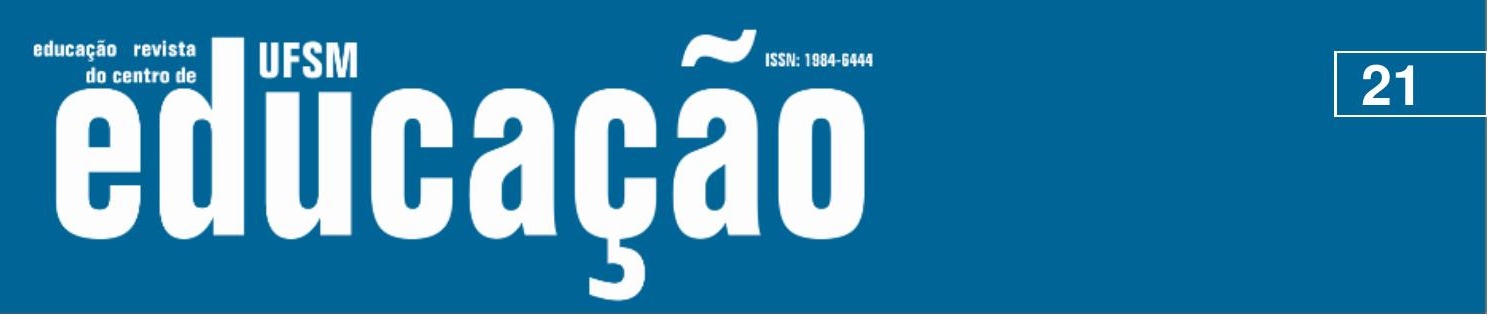

ISSN: 1984-6444 | http://dx.doi.org/10.5902/1984644434888

ÁVALOS, B. Teacher regulatory forces and accountability policies in Chile: from public servants to accountable professionals. Research Papers in Education, v. 19, n. 1, p. 67-85, 2004.

ÁVALOS, B. Teacher evaluation in Chile: highlights and complexities in 13 of experience. Teachers and Teaching, v. 24, n. 3, p. 297-311, 2017. https://doi.org/10.1080/13540602.2017.1388228

ÁVALOS, B.; ASSAÉL, J. Moving from Resistance to Agreement: The Case of the Chilean Teacher Performance Evaluation. International Journal of Educational Research, n. 45, p. 254-266, 2006.

ÁVALOS, B.; SOTOMAYOR, C. Cómo ven su identidad los docentes chilenos. Perspectiva Educacional, Formación de Profesores, v. 51, n. 1, p. 57-86, 2012.

BALL, S. Capítulo VIII: La gestión tecnología moral. Un análisis nudista. In: BALL, S. (Comp.). Foucault y la educación. Disciplinas y saber. Madrid: Ediciones Morata, Fundación Paideia, 2001. p. 155-168.

BALL, S . Profesionalismo, Gerencialismo y Performatividad. Revista Educación y Pedagogía, v. 25, n. 37, p. 87-104, 2003.

BALL, S.; YOUDELL, D. La privatización encubierta en la educación pública. Londres: Universidad de Londres, 2008.

BELLEI, C.; VALENZUELA, J.P. ¿Están las Condiciones para que la Docencia sea una Profesión de Alto Estatus en Chile? En: MARTINIC, C.; ELACQUA, G. (Eds.).

Fin de ciclo Cambios en la gobernanza del sistema educativo. Santiago: UNESCO/PUC, 2010. p. 257-283.

BELLEI, C.; VALENZUELA, J.P. El estatus de la profesión docente en Chile. Percepción de los profesores acerca del status profesional de la docencia. En:

ÁVALOS, B. (Ed.). ¿Héroes o villanos? La profesión docente en Chile. Santiago: Editorial Universitaria, 2013. p. 175-206.

BISCARRA, C.; GIACONI, C.; ASSAÉL, J. El docente en la legislación educacional chilena. Psicoperspectivas, v. 14, n. 3, p. 80-92, 2015.

BONIFAZ, R. Capítulo 1: Origen de la Evaluación Docente y su conexión con las políticas públicas en educación. En: MANZI, J.; GONZÁLEZ, R.; SUN, Y. (Eds.). La evaluación docente en Chile. Santiago: MIDE UC, 2011. p. 13-32.

CABALIN, C. Neoliberal Education and Student Movements in Chile: inequalities and malaise. Policy Futures in Education, v. 10, n. 2, p. 219-228, 2012.

http://dx.doi.org/10.2304/pfie.2012.10.2.219

CABEZAS, V.; MEDEIROS, M. P.; INOSTROZA, D.; GÓMEZ, C.; LOYOLA, V.

Organización del tiempo docente y su relación con la satisfacción laboral: Evidencia para el caso chileno. Archivos Analíticos de Políticas Educativas, v. 25, n. 64, 


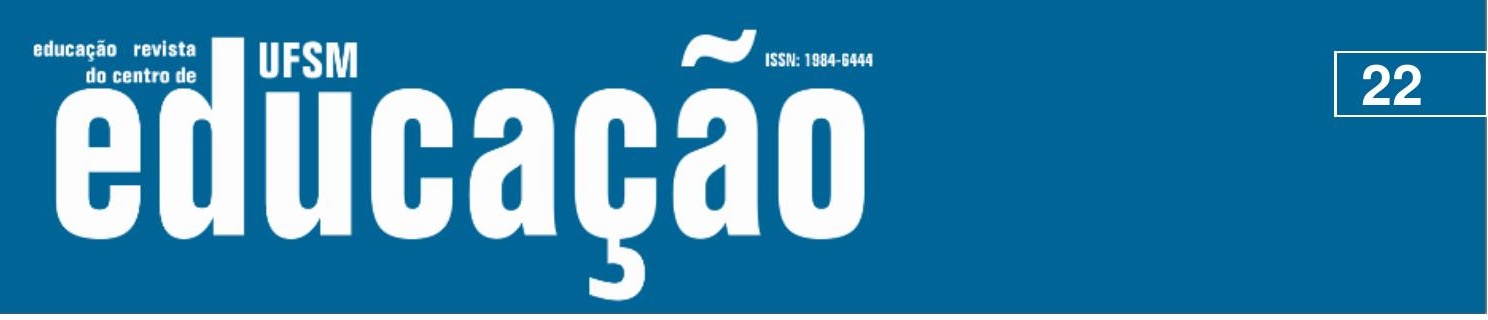

ISSN: 1984-6444 | http://dx.doi.org/10.5902/1984644434888

2017. http://dx.doi.org/10.14507/epaa.25.2451

CARRASCO, A. Mecanismos performativos de la institucionalidad educativa en Chile: Pasos hacia un nuevo sujeto cultural. Observatorio cultural, n. 15, p. 4-10, 2013.

CAVIERES, E.; APPLE, M. La ley docente y la clase media: Controlando el desarrollo de los profesores chilenos. Cadernos CEDES, v. 36, n. 100, p. 265-280, 2016.

CHILE. Ley n. 20.903, de 4 de marzo de 2016. Crea el Sistema de Desarrollo Profesional Docente y modifica otras normas. Biblioteca del Congreso Nacional de Chile. 2016. Disponible en: https://www.leychile.cl/Navegar?idNorma=1087343 . Acceso el: 17 jun. 2018.

COHEN, D. K.; MOFFITT, S. L. The ordeal of equality: Did federal regulation fix the schools? Cambridge: Harvard University Press, 2009.

CORNEJO, R. Condiciones de trabajo y bienestar/malestar docente en profesores de enseñanza media de Santiago de Chile. Educaçao \& Sociedade, v. 30, n. 107, p. 409-426, 2009.

CORNEJO, R . Repensar el trabajo docente en el chile actual: una necesidad para la Psicología Educacional. En: LEAL, F. (Ed.). Temas en Psicología Educacional: contribuciones para la formación de especialidad (en prensa). lquique:

Universidad de Tarapacá, 2017.

CORNEJO, R.; ALBORNOZ, N.; CASTAÑEDA, L.; PALACIOS, D.;

ETCHEBERRIGARAY, G.; FERNÁNDEZ, R.; GÓMEZ, S.; HIDALGO, F.; LAGOS, J. I. Las prescripciones del trabajo docente en el nuevo marco regulatorio de políticas educativas en Chile. Psicoperspectivas, v. 14, n. 2, p. 72-83, 2015.

CUADRA, D.; JORQUERA, R.; PÉREZ, M. Las teorías subjetivas del profesor acerca de su salud laboral: Implicancias en la promoción de la salud preventiva en el trabajo docente. Ciencia \& Trabajo, v. 17, n. 52, p. 1-6,

2015. https://dx.doi.org/10.4067/S0718-24492015000100002

ETCHEBERRIGARAY, G.; LAGOS, J.; CORNEJO, R.; ALBORNOZ, N.; FERNÁNDEZ, R. Concepciones sobre docencia en el nuevo marco regulatorio del sistema escolar chileno. Revista de Psicología, v. 26, n. 1, p. 1-13, 2017.

FALABELLA, A. El mercado escolar en Chile y el surgimiento de la Nueva Gestión Pública: el tejido de la política entre la dictadura neoliberal y los gobiernos de la centroizquierda (1979 a 2009). Educaçao \& Sociedade, v. 36, n. 132, p. 699-722, 2015.

FALABELLA, A . ¿Qué aseguran las políticas de aseguramiento de la calidad? Un estudio de casos en distintos contextos escolares. Estudios Pedagógicos, v. 42, n. 1, p. 107-126, 2016. http://dx.doi.org/10.4067/S0718-07052016000100007 


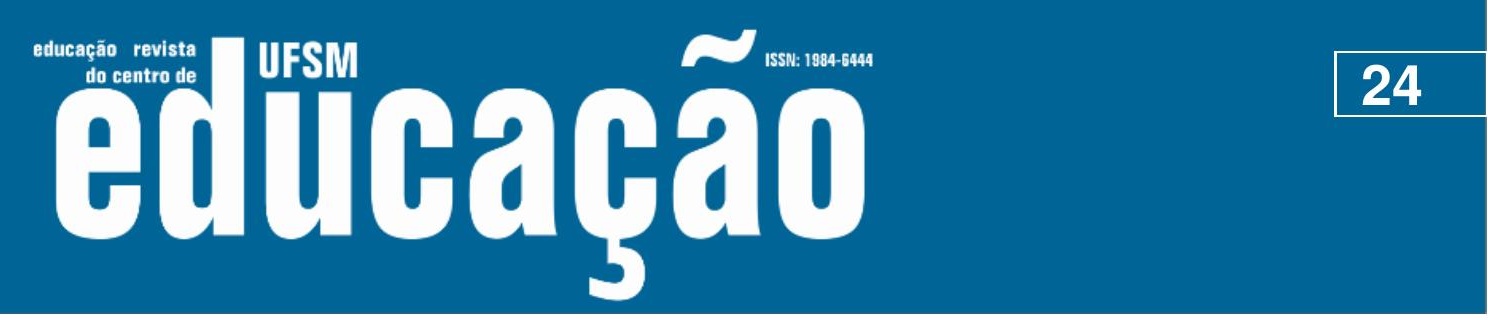

ISSN: 1984-6444 | http://dx.doi.org/10.5902/1984644434888

23, n. 88, 2015. http://dx.doi.org/10.14507/epaa.v23.2092

HALL, D.; GRIMALDI, E.; GUNTER, H.; MØLLER, J.; SERPIERI, R.; SKEDSMO, G. Educational reform and modernisation in Europe: The role of national contexts in mediating the new public management. European Educational Research Journal, v. 14 , n. 6 , p. $487-507,2015$.

HERR, K. Cultivating disruptive subjectivities: Interrupting the new professionalism.

Education Policy Analysis Archives, v. 23, n. 86, 2015.

http://dx.doi.org/10.14507/epaa.v23.2097

HOLLOWAY, J.; SØRENSEN, T. B.; VERGER, A. Global perspectives on highstakes teacher accountability policies: An introduction. Education Policy Analysis Archives, v. 25, n. 85, 2017. http://dx.doi.org/10.14507/epaa.25.3325

LARA, B.; MIZALA, A.; REPETTO, A. Una mirada a la efectividad de los profesores en Chile. Estudios Públicos, n. 120, p. 147-182, 2010.

LIGUEÑO, S.; PALACIOS, D.; SANDOVAL, J. Discursos de la vulnerabilidad bajo el gerenciamiento educativo: Análisis Ley de subvención escolar preferencial chilena. Revista de Investigación Psicológica, n. 19, p. 207-222, 2018.

MARCHESI, Á.; MARTÍN, E. Primera parte. Sociedad y Educación. Capítulo 1: La calidad de la enseñanza. En: MARCHESI, Á.; MARTíN, E. (Comp.). Calidad de la enseñanza en tiempos de cambio. Madrid: Alianza Editorial, 1998. p. 21-47.

MAROY, C.; PONS, X.; DUPUY, C. Vernacular globalisations: neo-statist accountability policies in France and Quebec education. Journal of Education Policy, v. 32, n. 1, p. 100-122, 2017.

https://doi.org/10.1080/02680939.2016.1239841

MEJÍA, M.R. Cambio curricular y despedagogización en la globalización, hacia una reconfiguración crítica de la pedagogía. Revista Docencia, n. 28, p. 40-53, 2006.

MEJÍA, M.R . La educación y la escuela: espacio en disputa de la reconfiguración capitalista. En: SIMPOSIO INTERNACIONAL DE EDUCACIÓN COMPARADA, 2016, Baja California, México. Instituto Internacional de Pedagogía Crítica Peter McLaren, 2016. p. 1-19.

MECKES, L.; BASCOPÉ, M. Uneven Distribution of Novice Teachers in the Chilean Primary School System. Education Policy Analysis Archives, v. 20, n. 30, 2012.

MONTECINOS, C.; PINO, M.; CAMPOS, J.; DOMÍNGUEZ, R.; CARREÑO, C. MASTER teachers as professional developers: Managing conflicting versions of professionalism. Educational Management Administration \& Leadership, v. 42, n. 2, p. 275-292, 2014.

MUÑOZ, M. L.; LÓPEZ, M.; ASSAÉL, J. Concepciones docentes para responder a la diversidad: ¿Barreras o recursos para la inclusión educativa? Psicoperspectivas, v. 


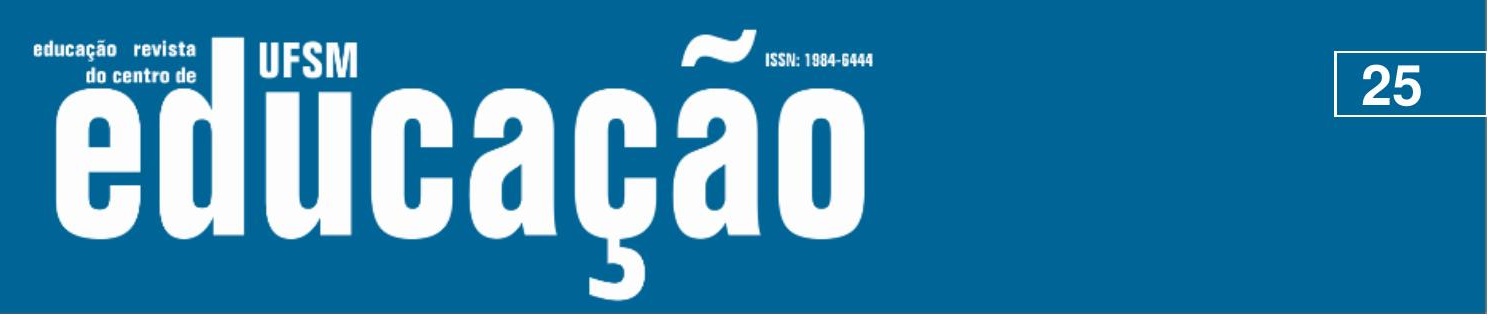

ISSN: 1984-6444 | http://dx.doi.org/10.5902/1984644434888

14, n. 3, p. 68-79, 2015.

NÚÑEZ, I. La profesión docente en Chile: saberes e identidades en su historia.

Revista Pensamiento Educativo, v. 41, n. 2, p. 149-164, 2007.

OBSERVATORIO CHILENO DE POLÍTICAS EDUCATIVAS (OPECH). Tensiones

de la Profesión Docente y sus Políticas de Desarrollo. En Documentos de trabajo OPECH. Disponible en: http://www.opech.cl/Libros/doc1.pdf. Acceso en: 4jun. 2018.

ORGANIZACIÓN PARA LA COOPERACIÓN Y EL DESARROLLO ECONÓMICO (OCDE). Los docentes son importantes: atraer, formar y conservar a los docentes eficientes. Paris: OCDE, 2009.

ORGANIZACIÓN PARA LA COOPERACIÓN Y EL DESARROLLO ECONÓMICO (OCDE). Capítulo 3: Fortalecer la calidad de la enseñanza y el liderazgo escolar en Chile. En: EVALUACIONES DE POLÍTICAS NACIONALES DE EDUCACIÓN. Educación en Chile. Santiago: OCDE, Fundación SM, 2017. p. 131-171.

OLESEN, K.G.; HASLE, P.; SØRENSEN, O. Social capital practices as adaptive drivers for local adjustment of new public management in schools. School Leadership \& Management, v. 36, n. 3, p. 333-352, 2016.

PARCERISA, L.; FALABELLA, A. La consolidación del Estado evaluador a través de políticas de rendición de cuentas: Trayectoria, producción y tensiones en el sistema educativo chileno. Education Policy Analysis Archives, v. 25, n. 89, 2017.

http://dx.doi.org/10.14507/epaa.25.3177

PAWSON, R.; GREENHALGH, T.; HARVEY, G.; WALSHE, K. Realist review - a new method of systematic review designed for complex policy interventions. Journal of Health Services Research \& Policy, v. 10, n. 1, p. 21-34, 2005.

PINO, M.; OYARZÚN, G.; SALINAS, I. Crítica a la estandarización para la rendición de cuentas: narrativas de resistencia para transformar el sistema de evaluación en Chile. Cadernos CEDES, v. 36, n. 100, p. 337-354, 2016.

https://doi.org/10.1590/cc0101-32622016171362

QUARESMA; M.; ORELLANA, V. El accountability y su impacto en la labor docente: percepciones de los profesores de liceos públicos de alto rendimiento académico en Chile. Currículo sem Fronteiras, v. 16, n. 2, p. 316-338, 2016.

SISTO, V. Nuevo profesionalismo y profesores: una reflexión a partir del análisis de las actuales políticas de 'profesionalización' para la educación en Chile. Signo y Pensamiento, v. 31, n. 59, p. 178-192, 2011.

SISTO, $V$. Identidades desafiadas: individualización, managerialismo y trabajo docente en el Chile actual. PSYKHE, v. 21, n. 2, p. 35-46, 2012.

SISTO, V.; FARDELLA, C. Nuevas políticas públicas, epocalismo e identidad: el caso de las políticas orientadas a los docentes en chile. Revista de Estudios 


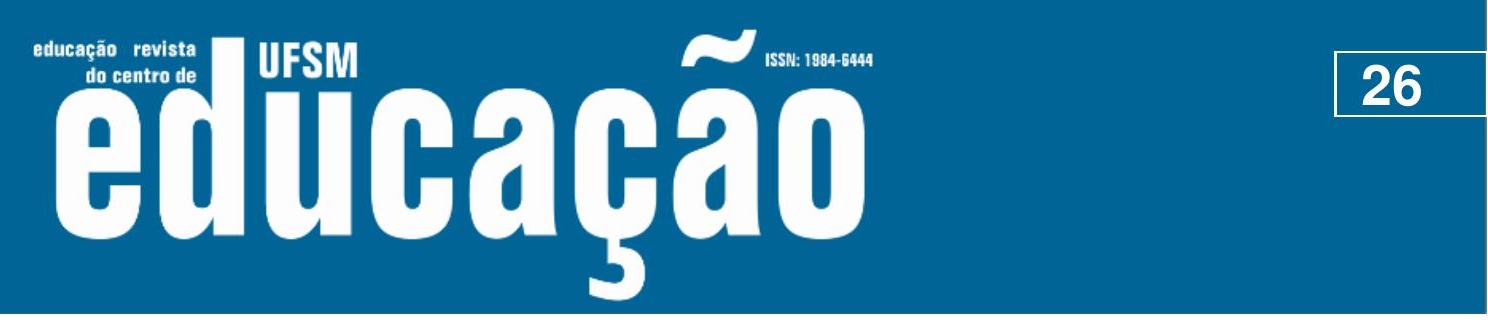

ISSN: 1984-6444 | http://dx.doi.org/10.5902/1984644434888

Universitários, v. 37, n. 1, p. 123-141, 2011.

SISTO, V.; FARDELLA, C . El eclipse del profesionalismo en la era de la Rendición de Cuentas. Modelando Docentes en el Contexto del Nuevo Management Público. Cadernos de Educação, n. 49, p. 3-23, 2014.

SISTO, V.; MONTECINOS, C.; AHUMADA, L. 2013. Disputas de significado e identidad: la construcción local del trabajo docente en el contexto de las políticas de evaluación e incentivo al desempeño en Chile. Universitas Psychologica, v. 12, n. 1, p. 173-184, 2013.

SOTO, R.; MERA, J.M.; NUÑEZ, C.; SISTO, V.; FARDELLA, C. Entre la efectividad y los afectos: nuevos docentes en tiempos de Nuevo Management Público. Athenea Digital. Revista de Pensamiento e Investigación Social, v. 16, n. 3, p. 3-19, 2016.

TAUT, S.; SUN, Y. The Development and Implementation of a National, Standardsbased, Multi-method Teacher Performance Assessment System in Chile. Education Policy Analysis Archives, v. 22, n. 71, 2014.

http://dx.doi.org/10.14507/epaa.v22n71.2014

TAUT, S.; SANTELICES, V.; ARAYA, C.; MANZI, J. Theory underlying a national teacher evaluation program. Evaluation and Program Planning, v. 33, n. 4, p. 477486, 2011. https://doi.org/10.1016/j.evalprogplan.2010.01.002

TSANG, K.K.; KWONG, T. L. Teachers' emotions in the context of education reform: labor process theory and social constructionism. British Journal of Sociology of Education, v. 38, n. 6, p. 841-855, 2016.

https://doi.org/10.1080/01425692.2016.1182007

REYES, I.; AKKARI, A. La privatización de la educación en Chile: análisis de los discursos del profesorado y de la dirección de los centros sobre la calidad de la educación y la rendición de cuentas. Revista de la Asociación de Sociología de la Educación, v. 10, n. 3, p. 363-380, 2017.

REYES, L.; CORNEJO, R.; ARÉVALO, A.; SÁNCHEZ, R. Ser docente y subjetividad histórica en el Chile actual: discursos, prácticas y resistencias. Polis, v. 9, n. 27, 269292, 2010.

RIVERO, R. The link of teacher career paths on the distribution of high qualified teachers: A Chilean case study. Archivos Analíticos de Políticas Educativas, v. 23, n. 73, 2015. http://dx.doi.org/10.14507/epaa.v23.1710

ROJAS, M. T.; LEYTON, D. The new teacher subjectivity: Construction of teachers' subjectivities at the beginning of the Preferential School Voucher's implementation in Chile. Estudios Pedagógicos, 40, número especial, p. 205-221, 2014. https://dx.doi.org/10.4067/S0718-07052014000200012

RUFFINELLI, A. Dificultades de la iniciación docente: ¿iguales para todos? Estudios 


\section{educação revista আ IISM \\ ISSN: 1984-6444

ISSN: 1984-6444 | http://dx.doi.org/10.5902/1984644434888

Pedagógicos, v. 40, n. 1, p. 229-242, 2014. https://dx.doi.org/10.4067/S0718$\underline{07052014000100014}$

RUFFINELLI, A . Ley de desarrollo profesional docente en Chile: de la precarización sistemática a los logros, avances y desafíos pendientes para la profesionalización. Estudios Pedagógicos, v. 42, n. 4, p. 261-279, 2016. https://dx.doi.org/10.4067/S0718-07052016000500015

RUFFINELLI, A.; GUERRERO, A. Círculo de segmentación del sistema educativo chileno: Destino laboral de egresados de pedagogía en educación básica. Revista Calidad en la Educación, n. 31, p. 20-44, 2009.

SANTORI, D. Public narratives under intensified market conditions: Chile as a critical case. Discourse: Studies in the Cultural Politics of Education, v. 39, n. 1, p. 5368, 2018. https://doi.org/10.1080/01596306.2016.1229267

VERGER, A.; NORMAND, R. Nueva Gestión Pública y educación: elementos teóricos y conceptuales para el estudio de un modelo de reforma educativa global. Educaçao \& Sociedade, v. 36, n. 132, p. 599-622, 2015.

VERGER, A.; ZANCAJO, A.; FONTDEVILA, C. La economía política de la privatización educativa: políticas, tendencias y trayectorias desde una perspectiva. Revista Colombiana de Educación, v. 70, p. 47-78, 2016.

WEINSTEIN, J.; MARFÁN, J.; HORN, A.; MUÑOZ, G. Chile: School leadership challenged by double accountability towards school. In: EASLY II, J.; TULOWITZKI, P. (Eds.). Educational Accountability. International perspectives on challenges and possibilities for school leadership. New York: Taylor \& Francis Group, 2016. p. 54-72.

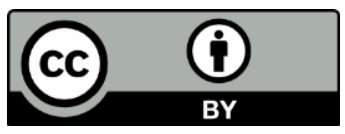

This work is licensed under a Creative Commons Attribution 4.0 International (CC BY 4.0). 